MODELING, IDENTIFICATION AND CONTROL, 1981, VOL. 2, NO. 2, 107-118

doi: $10.4173 /$ mic.1981.2.5

\title{
On the use of a block analogue of the Gerschgorin circle theorem in the design of decentralized control of a class of large-scale systems
}

\author{
OLE A. SOLHEIM $\dagger$
}

Keywords: decentralized control, decentralized estimation, eigenvalues, stability, the block Gerschgorin theorem.

The paper deals with the design of decentralized control of interconnected dynamic systems. It is assumed that each subsystem has its own control input and that the interconnections are through the states of the other subsystems.

The purpose of the present paper is to investigate the possibility of using the so-called block Gerschgorin theorem to evaluate the stability of the total system, given the local controllers. This theorem enables us to determine inclusion regions for the eigenvalues of the total system and these regions are usually sharper than those obtained by the usual Gerschgorin circle theorem.

\section{Introduction}

In this paper we consider control of interconnected dynamic systems. We assume that the total system consists of $N$ subsystems described by

$$
\dot{x}_{i}=A_{i i} x_{i}+\sum_{\substack{j=1 \\ j \neq i}}^{N} A_{i j} x_{j}+B_{i} u_{i}, \quad i=1,2, \ldots, N
$$

where $x_{i}$ is an $n_{i}$-dimensional state vector $\left(\sum_{i=1}^{N} n_{l}=n\right)$ and $u_{i}$ an $m_{i}$-dimensional control vector $\left(\sum_{i=1}^{N} m_{t}=m\right)$. We note that each subsystem has its own control input and that the interconnections are through the states of the other subsystems.

In this paper, we shall discuss decentralized control, that is, we consider control laws of the form

$$
u_{i}=G_{i} x_{i}
$$

Such a control scheme is advantageous in the sense that each local control station uses information from its own subsystem only. On the other hand, there are some drawbacks concerning overall performance. If, for example, optimal control is considered, the performance of a system with decentralized control will usually be inferior to a system with centralized control. However, the most serious problem we are faced with, when using decentralized control in the form of the control law (2), is probably the question of stability of the overall system.

In the present paper, we shall deal with this last problem only. For the subsequent discussion it is immaterial how the local controllers $G_{i}$ have been determined.

Received 15 October 1980.

$\uparrow$ The University of Trondheim, The Norwegian Institute of Technology, Division of Engineering Cybernetics, 7034 Trondheim-NTH. 
The problem we are faced with is, thus, the evaluate the stability of the overall system, given the local controllers. This question has received much attention recently. An extensive bibliography is, for example, given by Sandell et al. (1978).

To evaluate the overall stability we may use, for example, the eigenvalue location. One method presented by Sundareshan (1977) (see also Singh and Titli (1978)), is based on cxponential stabilization with a prescribed degree $\alpha$ of the overall system (all eigenvalues have real parts less than $-\alpha$ ).

The purpose of the present paper is to investigate the possibility of using the so-called block Gerschgorin theorem to evaluate the overall stability. This theorem gives inclusion regions for the eigenvalues of the overall system. These inclusion regions are usually sharper than those obtained by the usual Gerschgorin circle theorem.

The advantage of using this block Gerschgorin in the stability study is that the necessary computations are rather simple and that variations in parameters, and to some extent also in structure, of the interconnections can be studied without much extra computational effort.

The main drawback of the method is that the inclusion regions in general are not very sharp, that is, the stability will usually be better than the method indicates. This may, however, not always be a drawback, when one considers the design, since it will result in a safe (conservative) design.

With all the local controllers given, the eigenvalues of the overall system may, of course, be computed directly. It is, however, not so easy to evaluate the influence on stability of parameter and structure variations of the interconnections in this way. It is probable here that the block Gerschgorin has its greatest potential value.

The material in the present paper is arranged in the following way. In $\S 2$ we present the block Gerschgorin and some related theorems. In $\$ 3$ are discussed some applications of the theorem in decentralized control systems, and in $\$ 4$ some applications in systems with decentralized state estimation. In $\S 5$ are given some numerical examples to illustrate the use of the block Gerschgorin theorem.

\section{The block Gerschgorin theorem}

The block Gerschgorin theorem, which is a generalization of the Gerschgorin circle theorem, was first presented by Feingold and Varga (1962). Many of the results relating to the circle theorem can also be generalized. Below, we state the main theorem and some related theorems. The proofs of the theorems will be omitted here but can be found in the above cited reference. Some more results relating to the block theorem can be found in Powers (1976), and in Kovarik and Olesky (1974).

Let $A$ be any $n \times n$ matrix with complex entries which is partitioned in the following manner:

$$
A=\left[\begin{array}{ccc}
A_{11} A_{12} & \ldots & A_{1 N} \\
A_{21} A_{22} & \ldots & A_{2 N} \\
\hdashline A_{N 1} A_{N 2} & \ldots & A_{N N}
\end{array}\right]
$$

where the diagonal submatrices $A_{i i}, i=1,2, \ldots, N$, are square of order $n_{i}$. 
Considering the rectangular matrix $A_{i j}$ for any $1 \leqslant i, j \leqslant N$ as a linear transformation from the $n_{j}$-dimensional vector subspace $\Omega_{j}$ to the $n_{i}$-dimensional vector subspace $\Omega_{i}$, the norm $\left\|A_{i j}\right\|$ is defined as

$$
\left\|A_{i j}\right\|=\sup _{\substack{x \neq 0 \\ x \in \Omega_{j}}} \frac{\left\|A_{i j} x\right\|_{\Omega_{i}}}{\|x\|_{\Omega_{j}}}
$$

where $\|x\|_{\Omega_{j}}$ is a vector norm associated with the subspace $\Omega_{j}$.

Note that if the partitioning in (3) is such that all matrices $A_{i j}$ are $1 \times 1$ matrices and $\|x\|_{\Omega_{i}} \equiv|x|$, then the norms $\left\|A_{i j}\right\|$ are just the moduli of the single entries of these matrices. As no confusion arises, we shall drop the subscripts on the different vector norms, except that we shall use the notation $\|x\|_{2}$ to denote the Euclidean norm $\|x\|_{2} \equiv\left[\sum_{i}\left(x_{i}\right)^{2}\right]^{1 / 2}$.

Now, using the $\|x\|_{2}$-norm, the norm (4) is equivalent to

$$
\left\|A_{i j}\right\|=\sqrt{ } \lambda_{\max }
$$

where $\lambda_{\max }$ is the largest eigenvalue of the matrix $A_{i j} A_{i j}{ }^{\mathrm{T}}$.

In the following, we also need the relation

$$
\left(\left\|A_{j j}{ }^{-1}\right\|\right)^{-1}=\inf _{\substack{x \neq 0 \\ x \in \Omega, j}} \frac{\left\|A_{j j} x\right\|}{\|x\|}
$$

where $A_{j j}$ is assumed to be non-singular. We can define $\left(\left\|A_{j j}{ }^{-1}\right\|\right)^{-1}$ to be zero whenever $A_{j j}$ is singular.

\section{Theorem 1 (Block Gerschgorin)}

For the partitioned matrix $A$ of (3), each eigenvalue of $A$ satisfies

$$
\left(\left\|\left(A_{j j}-\lambda I_{j}\right)^{-1}\right\|\right)^{-1} \leqslant \sum_{\substack{k=1 \\ k \neq j}}^{N}\left\|A_{j k \|}\right\|
$$

for at least one $j, 1 \leqslant j \leqslant N$.

In (7), $I_{j}$ denotes the $n_{j} \times n_{j}$ identity matrix.

It is worth while to note that if the partitioning of (3) is such that all the diagonal submatrices are $1 \times 1$ matrices and $\|x\|=|x|$, then Theorem 1 reduces to the usual Gerschgorin circle theorem.

In Theorem 1, we see that each eigenvalue $\lambda$ of an arbitrary $n \times n$ complex matrix $A$ necessarily satisfies (7) for at least one $j, 1 \leqslant j \leqslant N$. Now, for the partitioned matrix $A$ of (3), let the Gerschgorin set $F_{j}$ be the set of all complex numbers $z$ such that

$$
\left.\left(\| A_{j j}-z I_{j}\right)^{-1} \|\right)^{-1} \leqslant \sum_{\substack{k=1 \\ k \neq j}}^{N}\left\|A_{j k}\right\|, \quad 1 \leqslant j \leqslant N
$$

From (6) we then conclude that the Gerschgorin set $F_{j}$ always contains the eigenvalues of $A_{j j}$, independent of the magnitude of the right side of $(8)$ and independent 
of the vector norms used. According to Theorem 1, all the eigenvalues of $A$ lie in the set $F$ defined as

$$
F=\bigcup_{j=1}^{N} F_{j}
$$

The following theorem which is also a generalization of a familiar result of Gerschgorin, may be useful.

\section{Theorem 2}

If the union $H=\bigcup_{j=1}^{m} F_{p_{j}}, 1 \leqslant p_{j} \leqslant N$, of $m$ Gerschgorin sets is disjoint from the remaining $N-m$ Gerschgorin sets for the partitioned matrix $A$ of (3), then $H$ contains precisely $\sum_{j=1}^{m} n_{p_{j}}$ eigenvalues of $A$.

Going back to Theorem 1, the difficult point in applying this theorem is to develop the left side of (8). For our application here to the stability study of interconnected systems, we shall require that each Gerschgorin set $F_{J}$ consists of the union of circles. To this end, we use the following theorem:

\section{Theorem 3}

Let the partitioned matrix $A$ of (3) be such that its diagonal submatrices $A_{j J}$ are all normal ( $A$ matrix is called normal if it commutes with its conjugate transpose). If the Euclidean vector norms $\|x\|_{2}$ are used for each subspace $\Omega_{j}, 1 \leqslant j \leqslant N$, then each Gerschgorin set is the union of $n_{j}$ circles.

In view of (8), the centre of the $n_{j}$ circles are the eigenvalues of $A_{j j}$ and the radius is equal to the right side of (8).

We note that Theorem 3 puts rather severe restrictions on the diagonal submatrices. This point will be discussed in more detail in $\S 3$.

The last theorem we present here concerns the interchange of columns and rows in Theorem 1. Since the eigenvalues of $A$ and $A^{\mathrm{T}}$ are the same, we may replace column sums by row sums in the above theorems. Let us define

$$
R_{J} \equiv \sum_{\substack{k=1 \\ k \neq j}}^{N}\left\|A_{j k}\right\|, \quad C_{J} \equiv \sum_{\substack{k=1 \\ k \neq j}}^{N}\left\|A_{k J}\right\|, \quad 1 \leqslant j \leqslant N
$$

\section{Theorem 4}

For any $\alpha, 0 \leqslant \alpha \leqslant 1$, each eigenvalue $\lambda$ of $A$ satisfies

$$
\left(\left\|\left(A_{j j}-\lambda I_{j}\right)^{-1}\right\|\right)^{-1} \leqslant R_{j}^{\alpha} C_{j}^{1-\alpha}
$$

for at least one $j, 1 \leqslant j \leqslant N$.

\section{Stability of interconnected control systems}

Let us consider the system (1) with the decentralized controllers (2) and assume that all parameters are known. We would like to evaluate the stability of the overall system in the way that we determine inclusion regions for the eigenvalues. We saw in the previous paragraph that, in order that these inclusion regions may be easily determined, we require that they are unions of circles. This again requires that the 
diagonal submatrices, which here are $A_{i i}+B_{i} G_{i}, i=1,2, \ldots, N$, must all be normal. We can, however, hardly require that the designers of the local control systems come up with controllers that make these diagonal submatrices normal. It will, therefore, usually be necessary to modify the diagonal subsystems specially for the stability study.

In order to make the submatrices

$$
A^{\prime}{ }_{u}=A_{u}+B_{\imath} G_{t}
$$

normal, we shall here discuss two methods, one of which is general and one more specialized.

The general method is to diagonalize the submatrices:

$$
M_{u}{ }^{-1} A^{\prime}{ }_{u} M_{u}=\Lambda_{t}
$$

where $M_{i i}$ is an eigenvector matrix of $A^{\prime}{ }_{i i}$.

It is always possible to design the subsystems $A^{\prime}{ }_{i l}$ so that they can be diagonalized, and we define

$$
M=\left[\begin{array}{ccccc}
M_{11} & 0 & 0 & \ldots & 0 \\
0 & M_{22} & 0 & & 0 \\
\hdashline 0 & & & & M_{N N}
\end{array}\right]
$$

The total system becomes

$$
A=M^{-1} A M=\left[\begin{array}{c:c:c:c}
\Lambda_{1} & M_{11}^{-1} A_{12} M_{12} & \cdots & M_{11}^{-1} A_{1 N} M_{N N} \\
\hdashline M_{22}^{-1} A_{21} M_{11} & \Lambda_{2} & \cdots & \\
\hdashline & & & \\
\hdashline M_{N N}^{-1} A_{N 1} M_{11} & & \ldots & \Lambda_{N}
\end{array}\right]
$$

This being a similarity transformation, the eigenvalues are left invariant.

Since the eigenvalues of the subsystems are already known, the only computations left concern the norms of the different off-diagonal submatrices. Using the result from $\S 2$, the Gerschgorin sets $F_{J}$ can now be constructed.

To simplify the norm computations, we may use the fact that

$$
\|A B\| \leqslant\|A\|\|B\|
$$

This will, however, take place at the expense of some sharpness of the regions.

Another method to obtain normal submatrices is based on the fact that if, in a matrix, we multiply column number ' $i$ ' with a constant $k$ and row number ' $i$ ' with $1 / k$, then the eigenvalues remain invariant. The use of thts method is, however, rather limited, since the submatrices have to be of special form if it is to work. 
In $\S 5$ we shall illustrate the two methods presented here by means of numerical examples.

\section{Decentralized state estimation}

If all the states of the different subsystems are not directly measurable, a possible solution is to use local state estimators. (We assume that all subsystems are observable.)

The decentralized estimation problem has been studied by, for example, Siljak and Vukčević (1978). We shall here be concerned with the stability problem only, and not with the performance of this type of estimation.

Assume the local measurement vectors

$$
y_{i}=D_{i} x_{i}, \quad i=1,2, \ldots, N
$$

In order to estimate the states $x_{i}$ of each subsystem, we construct estimators of the form

$$
\hat{x}_{i}=A_{i l} \hat{x}_{i}+\sum_{\substack{j=1 \\ j \neq i}}^{N} A_{i j} \hat{x}_{j}+B_{i} u_{i}+K_{i}\left(D_{i} x_{i}-D_{i} \hat{x}_{i}\right), \quad i=1,2, \ldots, N
$$

where ${ }^{\wedge}$ denotes estimated quantities and $K_{t}$ is the estimator gain.

We shall not be concerned here with how the estimator gain is determined. We only assume that all gains $K_{i}$ are known.

The control law (2) is changed to

$$
u_{t}=G_{t} \hat{x}_{t}
$$

The total system with feedback and estimators now becomes

$$
\left[\begin{array}{c}
\dot{x}_{1} \\
\vdots \\
\vdots \\
\dot{x}_{N} \\
\hdashline \hat{\hat{x}}_{1} \\
\vdots \\
\vdots \\
\hat{\hat{x}}_{N}
\end{array}\right]=\left[\begin{array}{ccc:ccc}
A_{11} & \ldots & A_{1 N} & B_{1} G_{1} & \ldots & 0 \\
\hdashline A_{N 1} & \ldots & A_{N N} & 0 & \ldots & B_{N} G_{N} \\
\hdashline K_{1} D_{1} & \ldots & 0 & C_{11} & \ldots & A_{1 N} \\
\hdashline & \ldots & & & \\
\hdashline 0 & \ldots & K_{D} D_{N} & A_{N 1} & \ldots & C_{N N}
\end{array}\right]\left[\begin{array}{c}
x_{1} \\
\vdots \\
\vdots \\
x_{N} \\
\hdashline \hat{x}_{1} \\
\vdots \\
\vdots \\
\hat{x}_{N}
\end{array}\right]
$$

where $C_{i i}=A_{i t}-K_{i} D_{i}+B_{i} G_{i}$.

By applying the transformation

$$
\left[\begin{array}{c}
x_{1} \\
\vdots \\
x_{N} \\
\hdashline \Delta x_{1} \\
\vdots \\
\Delta x_{N}
\end{array}\right]=\left[\begin{array}{l:l}
I & 0 \\
& \\
\hdashline & \\
I & -I
\end{array}\right]\left[\begin{array}{c}
x_{1} \\
\vdots \\
x_{N} \\
\hdashline \hat{x}_{1} \\
\vdots \\
\hat{x}_{N}
\end{array}\right]
$$


to (18) we get

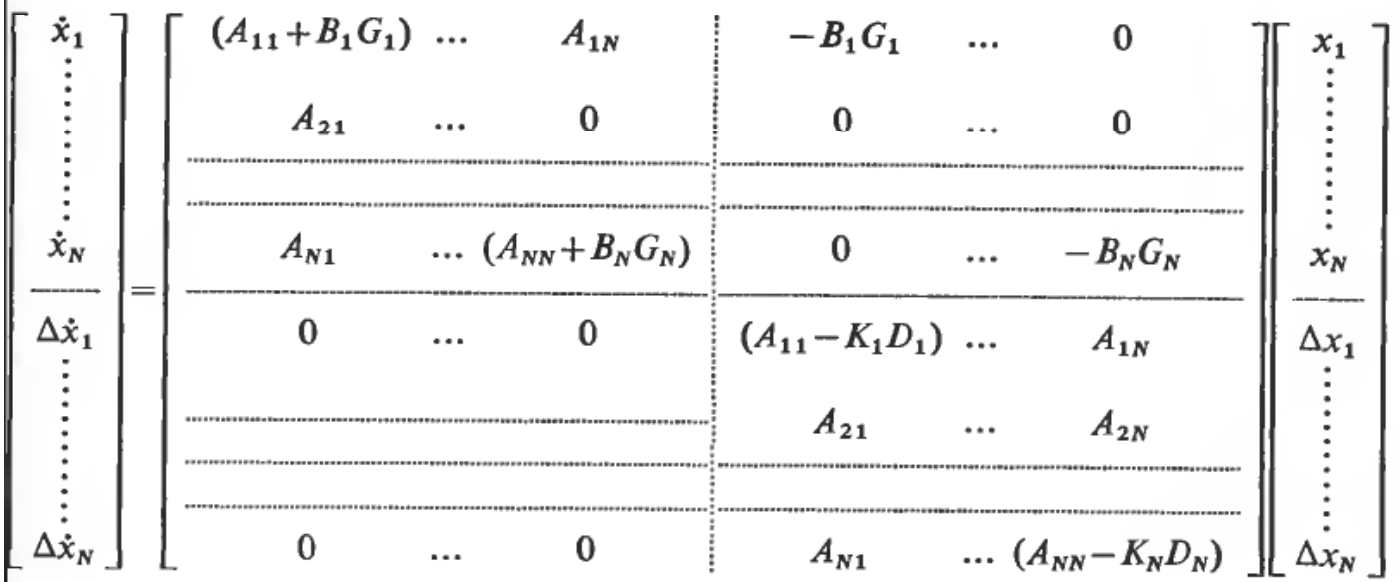

Thus, we have separated the eigenvalues of the feedback systems and the estimators. By using the methods of $\S 3$, we may now determine inclusion regions for all eigenvalues of (20).

We have assumed above, in the construction of the estimators, that the interconnections $A_{i j}$ were exactly known. If this is not the case, it is possible to evaluate the influence of inexactly known interconnections on stability by introducing the difference

$$
\Delta A_{i j}=A_{i j}-A_{i j}{ }^{\mathrm{j}}
$$

between the actual value of the interconnections and that used in the estimators. The system matrix in $(20)$ becomes now

$\left[\begin{array}{ccc:ccc}\left(A_{11}+B_{1} G_{1}\right) & \ldots & A_{1 N} & -B_{1} G_{1} & \ldots & 0 \\ \hdashline & & & & \\ \hdashline A_{N 1} & \ldots & \left(A_{N N}+B_{N} G_{N}\right) & 0 & \ldots & -B_{N} G_{N} \\ \hdashline 0 & \cdots & \Delta A_{1 N} & \left(A_{11}-K_{1} D_{1}\right) & \ldots & A_{1 N}{ }^{\mathrm{e}} \\ \hdashline & & & & & \\ \hdashline A_{N 1} & \ldots & 0 & A_{N 1}{ }^{\mathrm{e}} & \ldots & A_{N N}-K_{N} D_{N}\end{array}\right]$

We note that it is no longer possible to separate the eigenvalues but the methods of $\S 3$ can still be used to evaluate the stability. We note, for example, that any discrepancy in the actual value and the nominal value of the interconnections will increase the corresponding inclusion regions. 


\section{Numerical examples}

\section{Example 1}

Consider the following system with decentralized control:

$$
A^{1}=\left[\begin{array}{c:c}
A_{11}+B_{1} G_{1} & A_{12} \\
\hdashline A_{21} & A_{22}+B_{2} G_{2}
\end{array}\right]=\left[\begin{array}{rr:rr}
-4 & 2 & 1 & 0 \\
2 & -4 & 0 & 1 \\
\hdashline 1 & 0 & -4 & 2 \\
0 & 1 & 2 & -4
\end{array}\right]
$$

Using the vector norm $\|x\|_{2}$ we get $\left\|A_{12}\right\|=\left\|A_{21}\right\|=1$, and further

$$
\left.\left(|| A_{i l}-z I_{2}\right)^{-1} \|\right)^{-1}=\min \{|-6-z|,|-2-z|\}, \quad i=1,2
$$

The inclusion region $F_{i}$ thus consists of the points $z$ for which

$$
|-6-z| \leqslant 1, \quad|-2-z| \leqslant 1
$$

so that $F_{t}$ is the union of two disjoint circles, Fig. 1 .

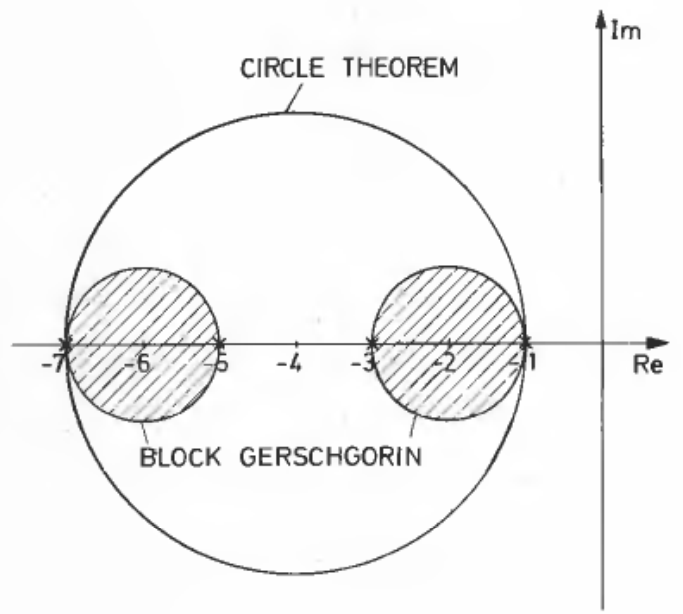

Figure 1.

The usual Gerschgorin circles for the matrix $A^{1}$ are all given by the single circle $|-4-z| \leqslant 3$, Fig. 1. We conclude that in this case the block Gerschgorin theorem gives significant improvement over the usual circle theorem.

The eigenvalues of the matrix $A^{1}$ are $-1,-3,-5,-7$. 


\section{Example 2}

Given the system

$$
A=\left[\begin{array}{rr:rr}
-2 & 0 & \multicolumn{2}{c}{A_{12}} \\
1 & -1 & & \\
\hdashline A_{21} & -2 & 0 \\
& & 1 & -1
\end{array}\right]
$$

Using the local controllers

$$
G_{t}=\left[\begin{array}{cc}
-3 \cdot 6 & -6 \cdot 0 \\
-12 \cdot 4 & -6 \cdot 4
\end{array}\right], \quad i=1,2
$$

the eigenvalues of the subsystems become $-5,-8$.

The total system becomes

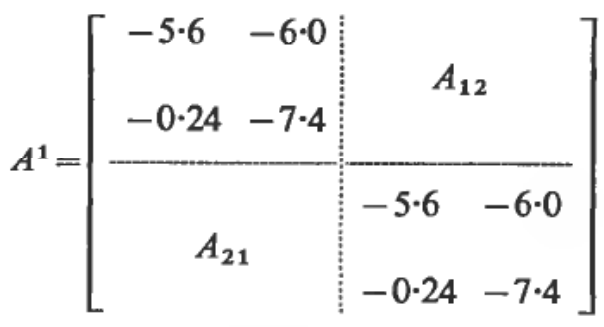

In order to make the diagonal submatrices normal, we multiply row 1 and row 3 with $0 \cdot 2$, and column 1 and 3 with 5 . Choosing

$$
A_{12}=A_{21}=\left[\begin{array}{ll}
0 & \epsilon_{1} \\
0 & 0
\end{array}\right]
$$

we get

$$
A^{1}=\left[\begin{array}{cc:cc}
-5.6 & -1.2 & 0 & 0.2 \epsilon_{1} \\
-1.2 & -7.4 & 0 & 0 \\
\hdashline 0 & 0.2 \epsilon_{1} & -5.6 & -1.2 \\
0 & 0 & -1.2 & -7.4
\end{array}\right]
$$

The inclusion regions $F_{i}, i=1,2$, consist of the union of circles with radius $0 \cdot 2 \epsilon_{1}$ and centres at -5 and -8 . This is illustrated in Fig. 2 with $\epsilon_{1}=5$. The eigenvalues of the total system are $-4 \cdot 64,-5 \cdot 48,-7 \cdot 52,-8 \cdot 36$.

Now we change the interconnections to

$$
A_{12}=A_{21}=\left[\begin{array}{ll}
0 & \epsilon_{1} \\
\epsilon_{2} & 0
\end{array}\right]
$$




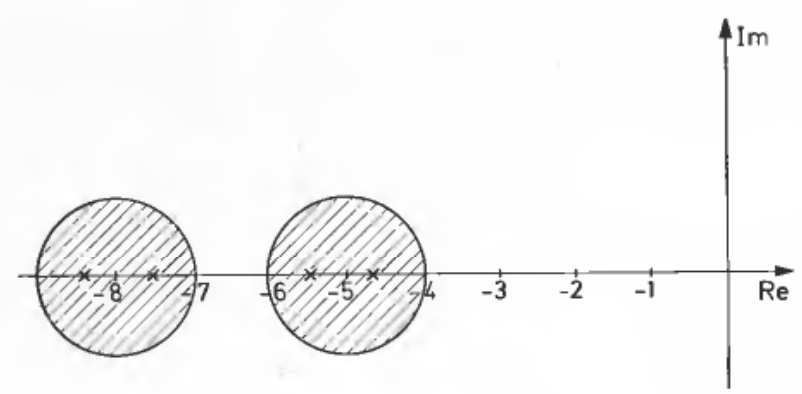

Figure 2.

The modified system (with normal diagonal submatrices) now becomes

$$
A^{1}=\left[\begin{array}{cc:cc}
-5.6 & -1.2 & 0 & 0.2 \epsilon_{1} \\
-1.2 & -7.4 & 5 \epsilon_{2} & 0 \\
\hdashline 0 & 0.2 \epsilon_{1} & -5.6 & -1.2 \\
5 \epsilon_{2} & 0 & -1.2 & -7.4
\end{array}\right]
$$

In this case the inclusion regions consist of circles with radius $\max \left\{\left|0 \cdot 2 \epsilon_{1}\right|,\left|5 \epsilon_{2}\right|\right\}$ and centres as before at -5 and -8 . We note that as long as $\epsilon_{2} \leqslant 0.04 \epsilon_{1}$, the inclusion regions are the same as in Fig. 2. With $\epsilon_{1}=5$ and $\epsilon_{2}=0 \cdot 2$, the eigenvalues of $A^{1}$ are $-4 \cdot 12,-5 \cdot 58,-7 \cdot 42,-8 \cdot 88$. See Fig. 3.

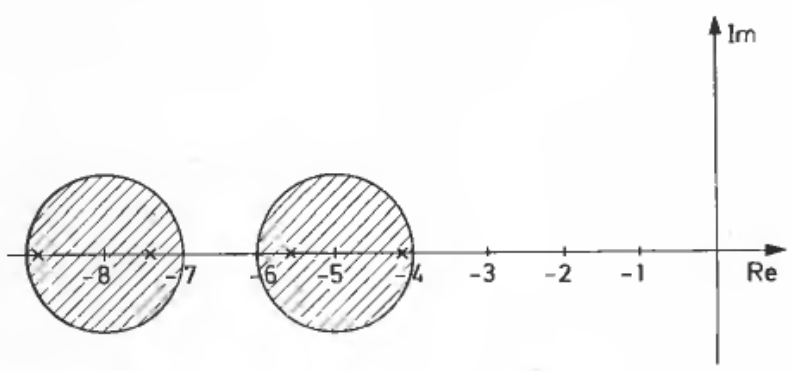

Figure 3.

\section{Example 3}

Given the following system with decentralized control:

$$
A^{1}=\left[\begin{array}{rr:rr}
-2 & -3 & 0 & \epsilon \\
2 & -7 & 0 & 0 \\
\hdashline 0 & \epsilon & -2 & -3 \\
0 & 0 & 2 & -7
\end{array}\right]
$$


In order to get normal diagonal submatrices, we may diagonalize each submatrix. Here the eigenvalues of the submatrices are -4 and -5 . Using the eigenvector matrix

$$
M_{11}=M_{22}=\left[\begin{array}{ll}
3 & 1 \\
2 & 1
\end{array}\right]
$$

we get (see eqns. (12) and (13))

$$
M^{-1} A^{1} M=\left[\begin{array}{rr:rr}
-4 & 0 & 2 \epsilon & \epsilon \\
0 & -5 & -4 \epsilon & -2 \epsilon \\
\hdashline 2 \epsilon & \epsilon & -4 & 0 \\
-4 \epsilon & -2 \epsilon & 0 & -5
\end{array}\right]
$$

The inclusion regions consist of the union of circles with radius $5 \epsilon$ and centres at -4 and -5 . This is illustrated in Fig. 4 where $\epsilon=0.2$. The eigenvalues are -3.69 , $-4 \cdot 5 \pm j \cdot 0 \cdot 39,-5 \cdot 31$.

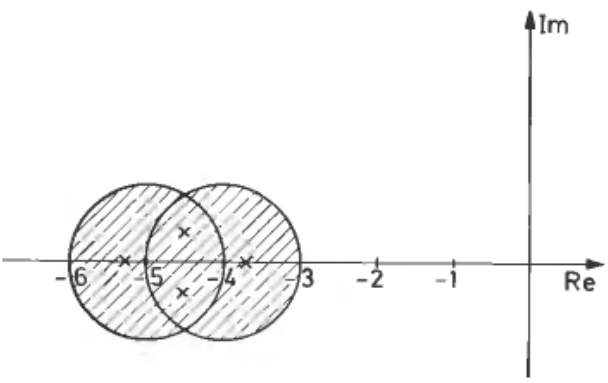

Figure 4.

\section{Concluding remarks}

It has been shown how the block Gerschgorin theorem, which is a generalization of the Gerschgorin circle theorem, can be used to study the stability of interconnected systems. The advantages of the method are that the necessary computations to be carried out are rather simple, and that variations in parameters and structure of the interconnections can be readily dealt with. The main drawback concerns the sharpness of the inclusion regions obtained by the method, and more investigations are required to clear up this point. The numerical examples presented in this paper indicates, however, that for the chosen systems the method gives a rather good evaluation of the stability.

\section{REFERENCES}

Feingold, D. G., and VARGA, R. S. (1962). Block diagonally dominant matrices and generalizations of the Gerschgorin circle theorem. Pacific J. Math., 12, 1241-1250.

KovariK, Z. V.; and Olesky, D. D. (1974). Sharpness of generalized Gerschgorin disks. Linear Algebra and its Applications, 8, 455-482. 
Powers, D. L. (1976). A block Gerschgorin theorem. Linear Algebra and its Applications, $13,45-52$.

Sandell, N. R. Jr., Varaiya, P., Athans, M., and Safonov, M. G. (1978). I.E.E.E. Trans. Autom. Control, 23, 108-128.

ŠILJAK, D. D., and VuKČEvić, M. B. (1978). On decentralized estimation. Int. J. Control, 27, 113-131.

Singh, M. G., and Titlı, A. (1978). Systems: Decomposition, Optimization and Control (Oxford: Pergamon Press).

SUNDARESHAN, M. K. (1977). Exponential stabilization of large-scale systems: Decentralized and multilevel schemes. I.E.E.E. Trans. Syst., Man and Cybern., 7, 478-483. 